\title{
Characterization and Construction of Permutation Graphs
}

\author{
Severino V. Gervacio ${ }^{1}$, Teofina A. Rapanut ${ }^{2}$, Phoebe Chloe F. Ramos ${ }^{2}$ \\ ${ }^{1}$ Department of Mathematics, De La Salle University, Manila, Philippines \\ ${ }^{2}$ Department of Mathematics and Computer Science, University of the Philippines, Baguio City, Philippines \\ Email: severino.gervacio@dlsu.edu.ph
}

Received September 15, 2012; revised October 15, 2012; accepted October 25, 2012

\begin{abstract}
If $\sigma$ is a permutation of $\{1,2, \cdots, n\}$, the graph $G_{\sigma}$ has vertices $1,2, \cdots, n$ where $x y$ is an edge of $G_{\sigma}$ if and only if $(x, y)$ or $(y, x)$ is an inversion of $\sigma$. Any graph isomorphic to $G_{\sigma}$ is called a permutation graph. In 1967 Gallai characterized permutation graphs in terms of forbidden induced subgraphs. In 1971 Pnueli, Lempel, and Even showed that a graph is a permutation graph if and only if both the graph and its complement have transitive orientations. In 2010 Limouzy characterized permutation graphs in terms of forbidden Seidel minors. In this paper, we characterize permutation graphs in terms of a cohesive order of its vertices. We show that only the caterpillars are permutation graphs among the trees. A simple method of constructing permutation graphs is also presented here.
\end{abstract}

Keywords: Permutation; Inversion; Permutation Graph; Cohesive Order; Oriented Graph; Tournament Score Sequence; Caterpillar; Graph Composition

\section{Introduction}

A bijection $\sigma$ of $\{1,2, \cdots, n\}$ to itself is called a permutation of order $n$. We shall write $\sigma=\left(a_{1}, a_{2}, \cdots, a_{n}\right)$ to mean that $\sigma(i)=a_{i}$ for $i=1,2, \cdots, n$. We shall denote by $\mathbb{S}_{n}$ the set of all permutations of $\{1,2, \cdots, n\}$. An inversion of $\sigma$ is an ordered pair $\left(a_{i}, a_{j}\right)$ where $i<j$ but $a_{i}>a_{j}$. Equivalently, $(x, y)$ is an inversion if and only if $x>y$ and $\sigma^{-1}(x)<\sigma^{-1}(y)$.

Definition 1.1 Let $\sigma \in \mathbb{S}_{n}$. The graph of inversions of $\sigma$, denoted by $G_{\sigma}$, is the graph with vertices $1,2, \cdots, n$ where $x y$ is an edge of $G_{\sigma}$ if and only if $(x, y)$ or $(y, x)$ is an inversion of $\sigma$.

The term graph of inversions was used by Ramos in [1]. For our purpose in this paper, any graph isomorphic to $G_{\sigma}$ for some permutation $\sigma$ will be called a permutation graph. There is an implementation PermutationGraph[p] in the Combinatorica package of Mathematica [2] that creates the permutation graph $G_{p}$.

In 1967 Gallai [3] characterized permutation graphs in terms of forbidden induced subgraphs. In 1971 Pnueli, Lempel, and Even [4] showed that a graph $G$ is a permutation graph if and only if both $G$ and its complement $\bar{G}$ have transitive orientations. Recently in 2010 Limouzy [5] gave a characterization of permutation graphs in terms of forbidden Seidel minors.

A characterization of permutation graphs in terms of cohesive vertex-set order is established in this paper. We prove that the only permutation graphs among the trees are the caterpillars. In addition, we describe a simple method of constructing permutation graphs.

\section{Cohesive Vertex-Set Order}

The vertex-set of a graph $G$ will be denoted by $V(G)$ while the edge-set will be denoted by $E(G)$. An edge with end-vertices $a$ and $b$ will be denoted by $a b$ or $b a$. For graph theoretic terms used here without definition, the book by Harary [6] may be referred to.

Consider the permutation $\sigma=(3,1,4,6,5,2)$. The inversions of $\sigma$ are $(3,1),(3,2),(4,2),(6,5)$, $(6,2)$, and $(5,2)$. It is convenient to draw the graph $G_{\sigma}$ with the vertices in a line following their arrangement in $\sigma$. A drawing of $G_{\sigma}$ is shown in Figure 1.

There are some important properties of the drawing that we need to take note of.

(a) If $a b$ and $b c$ are two edges of the graph where $b$ lies between $a$ and $c$ in the drawing, then $a c$ is also an edge.

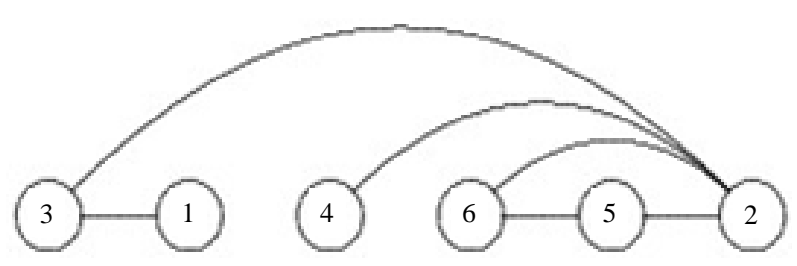

Figure 1. Permutation graph $G_{\sigma}, \sigma=(3,1,4,6,5,2)$. 
(b) If $u v$ is an edge and $x$ is a vertex that lies between $u$ and $v$ in the drawing, then either $u v$ is an edge or $x v$ is an edge.

We define more precisely the properties that we observed.

Definition 2.1 Let $G$ be a graph of order $n$. An arrangement $\ell=\left(v_{1}, v_{2}, \cdots, v_{n}\right)$ of the vertices of $G$ is called a cohesive vertex-set order of $G$ (or simply cohesive order $G$ ) if the following conditions are satisfied:

(a) If $i<k<j$ and $v_{i} v_{k}, v_{k} v_{j} \in E(G)$, then $v_{i} v_{j} \in E(G)$.

(b) If $i<k<j$ and $v_{i} v_{j} \in E(G)$, then $v_{k} v_{j} \in E(G)$ or $v_{k} v_{j} \in V(G)$.

The complement of a graph $G$, denoted by $\bar{G}$ has the same vertex-set as $G$ and two distinct vertices $a$ and $b$ form the edge $a b$ in $\bar{G}$ if and only if $a b$ is not an edge in $G$.

Lemma 2.1 Let $G$ be a graph. Then $\ell$ is $a$ cohesive order of $G$ if and only if $\ell$ is a cohesive order of $\bar{G}$.

Proof. Let $\ell=\left(v_{1}, v_{2}, \cdots, v_{n}\right)$ be a cohesive order of $G$. We claim that the same is a cohesive order of $\bar{G}$. To prove $(a)$ for $\bar{G}$, let $v_{i} v_{k}$ and $v_{k} v_{j}$ be vertices of $\bar{G}$ such that $i<k<j$. Then $v_{i} v_{k}$ and $v_{k} v_{j}$ are not edges in $G$. By property (b) of a cohesive order, the edge $v_{i} v_{j}$ is not in $G$. Hence, $v_{i} v_{j}$ is an edge of $\bar{G}$. To prove $(b)$ for $\bar{G}$, let $v_{i} v_{j}$ be an edge of $\bar{G}$ with $i<j$. Let $k$ be an integer such that $i<k<j$. Since $v_{i} v_{j}$ is in $\bar{G}$, then it is not in $G$. By property $(a)$ of a cohesive order (for $G$ ) the edges $v_{i} v_{k}$ and $v_{k} v_{j}$ cannot be both in $G$. Hence at least one of them is in $\bar{G}$.

The converse follows since $\overline{\bar{G}}=G$.

The next result follows easily from the definition of permutation graph and cohesive order. We shall omit the proof of this theorem.

Theorem 2.1 Let $\sigma \in \mathbb{S}_{n}$. Then

$$
\sigma=(\sigma(1), \sigma(2), \cdots, \sigma(n))
$$

is a cohesive order of the permutation graph $G_{\sigma}$.

Note that $\left(v_{1}, v_{2}, \cdots, v_{n}\right)$ is a cohesive order of a graph $G$ if and only if $\left(v_{n}, v_{n-1}, \cdots, v_{1}\right)$ is a cohesive order of G.

To assign a direction to an edge $a b$ of a graph $G$ means to change $a b$ to either the ordered pair $(a, b)$ or the ordered pair $(b, a)$.

Definition 2.2 An orientation of a graph $G$ is the digraph obtained by assigning a direction to each edge of $G$. The directed edges, which are ordered pairs, are called arcs.

A digraph $D$ is said to be transitive if $(x, z)$ is an arc of $D$ whenever $(x, y)$ and $(y, z)$ are arcs in $D$.

In a digraph $D$, the out-degree of a vertex $x$, denoted by $\operatorname{deg}_{D}^{+}(x)$ or simply $\operatorname{deg}^{+}(x)$ is the numnber of vertices $y$ in $D$ such that $(x, y)$ is an arc in
$D$. The in-degree of $x$, denoted by $\operatorname{deg}_{D}^{-}(x)$ or $\operatorname{deg}^{-}(x)$ is the number of vertices $z$ in $D$ such that $(z, x)$ is an arc in $D$.

An oriented complete graph is called a tournament [7]. The score of a vertex $x$ in a tournament, denoted by $s(x)$ is defined by $s(x)=\operatorname{deg}^{+}(x)$. The score sequence of a tournament is the sequence of scores arranged in non-decreasing order.

The following theorem [8] is not difficult, and is stated without proof.

Theorem 2.2 Let $T$ be a tournament of order $n$. The following statements are equivalent:

1) $T$ is transitive.

2) For all vertices $x$ and $y$ in $T$, if $(x, y)$ is an arc of $T$, then $s(x)>s(y)$.

3) For all vertices $x$ and $y$ in $T$, if $s(x)>s(y)$, then $(x, y)$ is an arc of $T$.

4) The score sequence of $T$ is $(0,1,2, \cdots, n-1)$.

Our main result, which characterizes permutation graphs, is the following theorem.

Theorem 2.3 A graph $G$ is a permutation graph if and only if it has a cohesive order.

Proof. If $G$ is a permutation graph, then $G$ is isomorphic to $G_{\sigma}$ for some permutation $\sigma$. By Theorem 2.1, $\sigma$ is a a cohesive order of $G_{\sigma}$. Let $\varphi$ be an isomorphism of $G$ to $G_{\sigma}$. Then $\alpha=\varphi^{-1} \circ \sigma$ is a cohesive order of $G$.

Conversely, let $G$ be a graph with a cohesive order $\alpha=\left(v_{1}, v_{2}, \cdots, v_{n}\right)$. Orient $G$ to obtain a digraph $D$ as follows: For each edge $v_{i} v_{j}$ in $G$, assign the direction $\left(v_{i}, v_{j}\right)$ if $i<j$; otherwise assign the direction $\left(v_{j}, v_{i}\right)$.

By property $(a)$ of a cohesive order, it follows that $D$ is transitive. Extend $D$ to a tournament by orienting the complement $\bar{G}$ of $G$ as follows: If $i<j$ but $v_{i} v_{j}$ is not in $D$, assign the direction $\left(v_{j}, v_{i}\right)$ to the edge $v_{i} v_{j}$ in $\bar{G}$. By Lemma $2.1 \alpha$ is a cohesive order of $\bar{G}$. So likewise, the orientation of $\bar{G}$ obtained in this manner is also transitive. Let us denote this digraph by $\bar{D}$.

The union of $D$ and $\bar{D}$ is an orientation of $G \cup \bar{G}$. Since $G \cup \bar{G}$ is complete, then $T=D \cup \bar{D}$ is a tournament. We claim that $T$ is a transitive tournament. Let $(x, y)$ and $(y, z)$ be arcs of $T$. If both arcs belong to $D$ or to $\bar{D}$, then $(x, z)$ is in $T$ because both $D$ and $\bar{D}$ are transitive. So let us assume that one of the arcs belong to $D$ and the other arc belong to $\bar{D}$. Without loss of generality, assume that $(x, y)$ is an arc in $D$, and $(y, z)$ is an arc in $\bar{D}$. If $(x, z)$ is in $D$, we are done. If $(x, z)$ is not in $D$, then $(z, x)$ is in $\bar{D}$. Since $D$ is transitive and $(z, x),(y, z)$ are in $\bar{D}$, then $(y, x)$ is in $\bar{D}$. This is a contradiction because $(x, y)$ is in $D$. 
By Theorem 2.2, the score sequence of $T$ is $(0,1, \cdots, n-1)$. Let $\sigma$ be the permutation defined by $\sigma(i)=1+s\left(v_{i}\right)$, where $s\left(v_{i}\right)$ is the score of $v_{i}$ in $T$. We claim that the mapping $\varphi: v_{1} \rightarrow 1+s\left(v_{i}\right)=\sigma(i)$ is an isomorphism of $G$ to $G_{\sigma}$.

The mapping $\varphi$ is bijective since the scores of the vertices are distinct. It remains to show that $\varphi$ preserves adjacency. Let $v_{i} v_{j}$ be an edge of $G$, where $i<j$. In $D$ we have the arc $\left(v_{i}, v_{j}\right)$. Since the tournament $T$ is transitive, then by Theorem 2.2, $s\left(v_{i}\right)>s\left(v_{j}\right)$. Hence, $(\sigma(i), \sigma(j))$ is an inversion of $\sigma$. Therefore, $\varphi\left(v_{i}\right)$ and $\varphi\left(v_{j}\right)$ are adjacent in $G_{\sigma}$. Conversely, let $a b$ be an edge in $G_{\sigma}$. Then either $(a, b)$ or $(b, a)$ is an inversion. Without loss of generality, assume that $(a, b)$ is an inversion. Let $a=\sigma(i)=1+s\left(v_{i}\right)$ and $b=\sigma(j)=1+s\left(v_{j}\right)$, where $i<j$. Since $(a, b)$ is an inversion, we have $a>b$, or $s\left(v_{i}\right)>s\left(v_{j}\right)$. Therefore, the arc $\left(v_{i}, v_{j}\right)$ is in $T$. Since $i<j$, the arc $\left(v_{i}, v_{j}\right)$ must be in $D$. Consequently, the edge $v_{i} v_{j}$ is in $G$.

Here is an illustration of the constructive proof of Theorem 2.3. Consider the graph $G$ shown in Figure 2 with a cohesive order $\left(x_{2}, x_{4}, x_{1}, x_{3}, x_{5}\right)$.

To be able to follow the discussion in the proof of theorem without difficulty, let

$$
\left(v_{1}, v_{2}, v_{3}, v_{4}, v_{5}\right)=\left(x_{2}, x_{4}, x_{1}, x_{3}, x_{5}\right) \text {. }
$$

Using the bottom drawing in Figure 2, we construct a digraph by directing all edges from left to right. For two vertices not adjacent in $G$, we assign the arc that goes from right to left. Then the result is a transitive tournament. It is not difficult to get the score of any vertex in this tournament. We simply count the eastbound arcs and the westbound arcs with a fixed tail. Consider for example, $v_{2}=x_{4}$. The number of eastbound arcs with tail at $x_{4}$ is 3 . The number of westbound arcs is simply the number of vertices to its left that are not adjacent to to $x_{4}$. The table below summarizes the scores of the vertices.

\begin{tabular}{cccccc}
\hline Vertex & $v_{1}=x_{2}$ & $v_{2}=x_{4}$ & $v_{3}=x_{1}$ & $v_{4}=x_{3}$ & $v_{5}=x_{5}$ \\
\hline Score, $\mathrm{s}\left(v_{\mathrm{i}}\right)$ & 2 & 4 & 0 & 1 & 3 \\
\hline
\end{tabular}

Take the permutation $\sigma$ defined by $\sigma(i)=1+s\left(v_{i}\right)$. Then $\sigma=(3,5,1,2,4)$. The permutation graph $G_{\sigma}$ is shown in Figure 3.

\section{Construction and Examples of Permutation Graphs}

Some fundamental facts about permutation graphs are given in the next theorem.

Theorem 3.1 Let $G$ be a graph. The following are equivalent:

(a) $G$ is a permutation graph.

(b) $\bar{G}$ is a permutation graph.

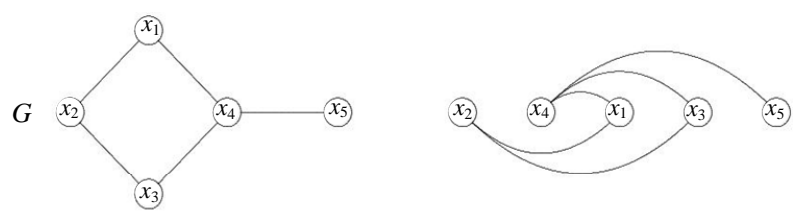

Figure 2. A graph $\boldsymbol{G}$ with cohesive order $\left(v_{1}, v_{2}, v_{3}, v_{4}, v_{5}\right)=\left(x_{2}, x_{4}, x_{1}, x_{3}, x_{5}\right)$.

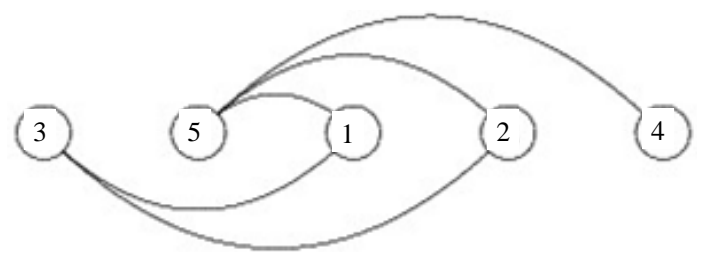

Figure 3. The permutation graph $\boldsymbol{G}_{\sigma}, \sigma=(3,5,1,2,4)$.

(c) Every induced subgraph of $G$ is a permutation graph.

(d) Every connected component of $G$ is a permutation graph.

Proof. From Lemma 2.1, $G$ has a cohesive order if and only if $\bar{G}$ has a cohesive order. Therefore, (a) and (b) are equivalent.

If $\left(v_{1}, v_{2}, \cdots, v_{n}\right)$ is a cohesive order of $G$, then the subgraph of $G$ induced by a set of vertices $\left\{v_{i_{1}}, v_{i_{2}}, \cdots, v_{i_{k}}\right\}$, where $i_{1}<i_{2}<\cdots<i_{k}$ has cohesive order $\left(v_{i_{1}}, v_{i_{2}}, \cdots, v_{i_{k}}\right)$ and therefore is a permutation graph. Hence, (a) and (c) are equivalent.

Statement (c) implies statement (d) because a connected component of $G$ is an induced subgraph of $G$.

It remains to show that (d) implies any of (a), (b), (c). Let $G$ have connected components $G_{1}, G_{2}, \cdots, G_{k}$ and let $n_{i}$ be the order of $G_{i}$. Let

$$
\ell_{i}=\left(v_{1}^{i}, v_{2}^{i}, \cdots, v_{n_{i}}^{i}\right)
$$

be a cohesive order of $G_{i}$. Then

$$
\begin{aligned}
& \ell=\left(\ell_{1}, \ell_{2}, \cdots, \ell_{k}\right) \\
& =\left(v_{1}^{1}, v_{2}^{1}, \cdots, v_{n_{1}}^{1}, v_{1}^{2}, v_{2}^{2}, \cdots, v_{n_{2}}^{2}, \ldots, v_{1}^{k}, v_{2}^{k}, \cdots, v_{n_{k}}^{k}\right)
\end{aligned}
$$

is a cohesive order of $G$. Therefore $G$ is a permutation graph.

We can now identify permutation graphs through the existence of a cohesive order. Moreover, we can even determine a permutation that generates a permutation graph isomorphic to the graph having a cohesive order.

Paths $P_{n}$ and stars $K_{1, n}$ are permutation graphs because they have cohesive orders as illustrated in Figure 4.

In the drawing of the path $P_{n}$, we have

$$
v_{1}=2, v_{2}=1, v_{3}=4, v_{4}=3, v_{5}=6 \text {, etc. }
$$

Condition (a) is vacuously satisfied because there is no 
pair of arcs $v_{i} v_{j}$, and $v_{j} v_{k}$ such that $i<j<k$. Note for example that $(2,3)$ is an arc and the vertices 1 and 4 are between 2 and 3 in the drawing. We have 1 adjacent to 2 and 4 adjacent to 3 . This illustrates condition (b).

In the drawing of the star $K_{1, n}$ we see that for every arc $(0, k)$ where $k>1$ all vertices $i$ with $0<i<k$ are between 0 and $k$. Moreover, the vertex $i$ is adjacent to 0 . Therefore condition (b) is satisfied. Condition (a) is satisfied vacuously.

Paths and stars are trees but not all trees are permutation graphs. Consider the tree $K_{1,3}^{*}$ formed by subdividing each edge of the star $K_{1,3}$ into two edges, as shown in Figure 5.

It is not difficult to argue indirectly that $K_{1,3}^{*}$ has no cohesive order. Therefore this is not a permutation graph. This result is also established by Limouzy [5] where he used the symbol $T_{2}$ for $K_{1,3}^{*}$.

Harary and Schwenk [9] defined a caterpillar to be a tree with the property that the removal of all pendant vertices results into a path. Figure 6 shows a caterpillar with 25 pendant vertices. The removal of these 25 pendant vertices yields the path $P_{8}$.

The next lemma is easy and its proof is omitted.

Lemma 3.1 A tree is a caterpillar if and only if it does not contain $K_{1,3}^{*}$ as a subgraph.

Theorem 3.2 A tree is a permutation graph if and only if it is a caterpillar.

Proof. A tree that contains $K_{1,3}^{*}$ is not a permutation

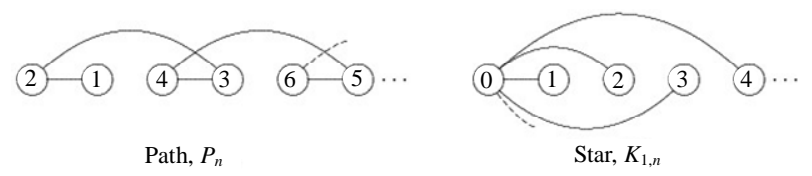

Figure 4. Cohesive order of paths and stars.

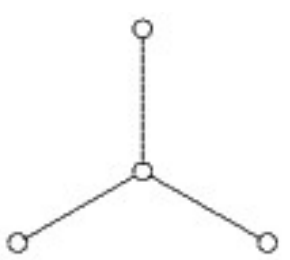

Star, $K_{1,3}$

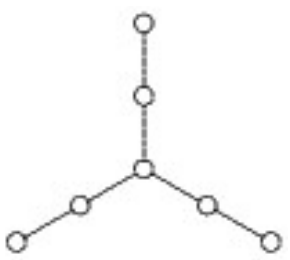

$K_{1,3}^{*}$
Figure 5. The tree $K_{1,3}^{*}$ obtained by subdividing the edges of $K_{1,3}$.

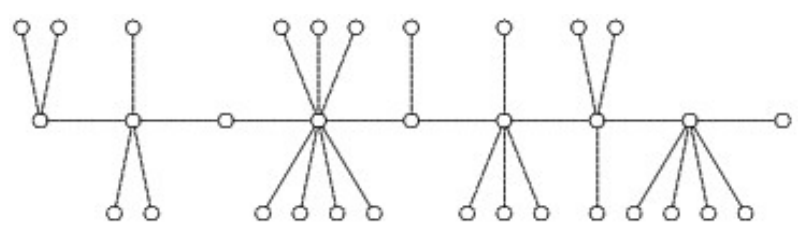

Figure 6. A caterpillar with 25 pendant vertices. graph because $K_{1,3}^{*}$ is not a permutation graph. Therefore, all we need to show is that a caterpillar is a permutation graph. Let $C$ be a caterpillar and let $P_{n}$ be the path obtained from $C$ by removing the pendant vertices. If $n=1$, then $C$ is either the trivial graph or the star $K_{1, p}$ for some $p \geq 1$. Since the trivial graph and the stars are permutation graphs, we assume that $n \geq 2$.

Let us form the cohesive order of $P_{n}$ as shown in Figure 4. Let $S_{i}$ be a set of pendant vertices of $C$ all adjacent to the same vertex $i$ of $P_{n}$. If $i$ is odd, we insert the vertices in $S_{i}$ immediately to the left of the vertex $i+1$ of the path (see Figure 4). If $i$ is even we insert the vertices in $S_{i}$ between $i$ and $i-1$. The result is a cohesive order of $C$. Therefore $C$ is a permutation graph.

Definition 3.1 Let $G$ be a graph with vertices $x_{1}, x_{2}, \cdots, x_{n}$ and let $H_{1}, H_{2}, \cdots, H_{n}$ be a collection of arbitrary graphs. The composition by $G$ of

$H_{1}, H_{2}, \cdots, H_{n}$, denoted by $G\left(H_{1}, H_{2}, \cdots, H_{n}\right)$ is the graph formed by taking the disjoint union of the graphs $H_{i}$ and then adding all edges of the form $a_{i} b_{j}$ where $a_{i}$ is in $H_{i}, b_{j}$ is in $H_{j}$ whenever $x_{i} x_{j}$ is an edge of $G$.

If each $H_{i}$ is equal to a fixed graph $H$, we use the symbol $G(H)$ to denote the composition.

The sum of two graphs $L$ and $M$, denoted by $L+M$ is formed by taking the disjoint union of $L$ and $M$ and then adding all edges of the form $a b$ where $a \in V(L)$ and $b \in V(M)$. Thus, the composition $G\left(H_{1}, H_{2}, \cdots, H_{n}\right)$ is formed by taking the disjoint union of the graphs $H_{i}$ and then forming the sum $H_{i}+H_{j}$ if the associated vertices $x_{i}$ and $x_{j}$ of $G$ are adjacent.

Theorem 3.3 Let $G$ be a graph of order $n$ and let $H_{1}, H_{2}, \cdots, H_{n}$ be arbitrary graphs. Then

$$
G\left(H_{1}, H_{2}, \cdots, H_{n}\right)
$$

is a permutation graph if and only if $G, H_{1}, H_{2}, \cdots, H_{n}$ are permutation graphs.

Proof. First, assume that $G\left(H_{1}, H_{2}, \cdots, H_{n}\right)$ is a permutation graph. Each graph $H_{i}$ is an induced subgraph of $G\left(H_{1}, H_{2}, \cdots, H_{n}\right)$. Therefore, each $H_{i}$ is a permutation graph. If we take a vertex $x_{i}$ from each $H_{i}$, then the subgraph induced by these vertices is isomorphic to $G$. Therefore $G$ is a permutation graph.

Conversely, assume that $G, H_{1}, H_{2}, \cdots, H_{n}$ are all permutation graphs. Then there is a cohesive order $\left(v_{1}, v_{2}, \cdots, v_{n}\right)$ of $G$. Let $n_{i}$ be the order of $H_{i}$. Then the vertices of $H_{i}$ has a cohesive order

$$
\ell_{i}=\left(x_{1}^{i}, x_{2}^{i}, \cdots, x_{n_{i}}^{i}\right) \text {. }
$$

It is easy to check that $\ell=\left(\ell_{1}, \ell_{2}, \cdots, \ell_{n}\right)$ is a cohesive order of $G\left(H_{1}, H_{2}, \cdots, H_{n}\right)$. 
Theorem 3.3 actually gives us an easy way of constructing permutation graphs by composition. To illustrate this, let $G$ be the star $K_{1,3}$ with central vertex $x_{1}$ and pendant vertices $x_{2}, x_{3}, x_{4}$, then

$$
G\left(P_{2}, C_{3}, P_{1}, P_{3}\right)
$$

is shown in Figure 7.

All graphs of order at most 4 are permutation graphs [1]. Therefore, $G\left(P_{2}, C_{3}, P_{1}, P_{3}\right)$ is a permutation graph.

Every graph $G$ of order $n$ may be written as

$$
G=G(\overbrace{P_{1}, P_{1}, \cdots, P_{1}}^{n}) \text { and } G=K_{1}(G) .
$$

If these are the only ways $G$ can be written as a composition, then we say that $G$ is prime.

It is easy to see that among the complete graphs, only $K_{1}$ and $K_{2}$ are prime permutation graphs.

Among trees with diameter not exceeding 3, it is easy to check that only the paths $P_{1}, P_{2}$, and $P_{4}$ are prime permutation graphs. These are all caterpillars that do not have two pendant vertices adjacent to a common vertex. Note that $P_{3}$ which is excluded from the list is a caterpillar with two pendant vertices having a common neighbor.

Theorem 3.4 A tree is a prime permutation graph if and only if it is a caterpillar where no two pendant vertices have a common neighbor.

Proof. In view of our observation about trees with diameter not exceeding 3 , we assume throughout that $T$ has diameter at least 4.

Let $T$ be a tree of order $n$. Assume that $T$ is a prime permutation graph. By Theorem 3.2 $T$ is a caterpillar. Suppose that $x_{1}$ and $x_{2}$ are pendant vertices with a common neighbor $y$. Let $G$ be the tree obtained from $T$ by identifying $x_{1}$ and $x_{2}$. Let

$y_{1}, y_{2}, \cdots, y_{n-1}$ be the vertices of $G$ Without loss of generality, assume that $y_{1}$ is the vertex resulting from the identification of $x_{1}$ and $x_{2}$. Let $H_{1}$ be the graph with two vertices but without an edge, and let $H_{i}$ be the trivial graph for $i=2,3, \cdots, n-1$. Then

$$
T=G\left(H_{1}, H_{2}, \cdots, H_{n}\right) .
$$

This contradicts the fact that $T$ is prime.

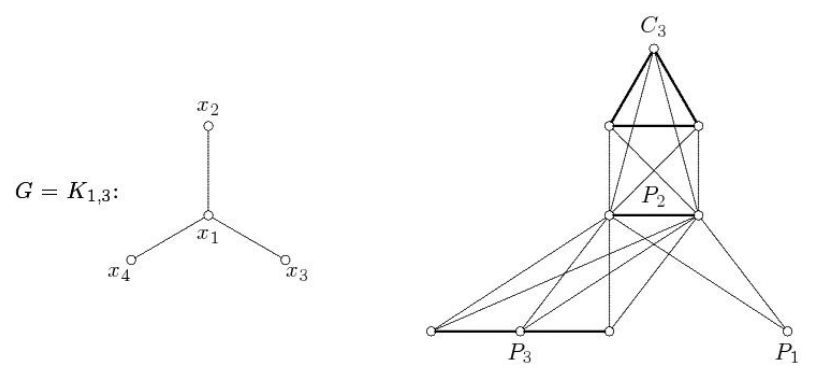

Figure 7. The composition by $K_{1,3}$ of $P_{2}, C_{3}, P_{1}, P_{3}$.
Conversely, assume that $T$ is a caterpillar with no two pendant vertices having a common neighbor. Suppose that $T$ is a not a prime permutation graph. Then for some non-trivial graph $G$ with vertices

$$
y_{1}, y_{2}, \cdots, y_{k}, T=G\left(H_{1}, H_{2}, \cdots, H_{k}\right) .
$$

Without loss of generality, we may assume that $H_{1}$ contains at least two vertices. Now, $G$ must be connected for otherwise, $T$ is disconnected. Let $y_{1}$ be adjacent to $y_{2}$ without loss of generality. Then

$$
H_{1}+H_{2}
$$

is a subgraph of $T$. If $H_{2}$ has at least two vertices, then there will be a cycle in $H_{1}+H_{2}$. Therefore, $H_{2}$ has only one vertex. In $G, y_{1}$ cannot be adjacent anymore to any other vertex for otherwise, we would also create a cycle of length 4 . Now consider $H_{1}+H_{2}$. There cannot be adjacent vertices in $H_{1}$ for otherwise we will create a cycle of length 3 . But then all vertices in $H_{1}$ are pendant vertices of $T$ and they have a common neighbor, the vertex in $H_{2}$. This is a contradiction.

Theorem 3.5 Let $G$ be a decomposable permutation graph. Then there exists a non-trivial prime permutation graph $U$ and permutation graphs

$$
H_{1}, H_{2}, \cdots, H_{k}
$$

which are subgraphs of $G$ such that

$$
G=U\left(H_{1}, H_{2}, \cdots, H_{k}\right) .
$$

Proof. Let

$$
G=U\left(H_{1}, H_{2}, \cdots, H_{k}\right)
$$

be a decomposition of $G$, where $U$ is non-trivial. If we take one vertex $x_{i}$ from each $H_{i}$, then the subgraph induced by these vertices is isomorphic to $U$. Hence, $U$ must be a permutation graph. Each $H_{i}$ is an induced subgraph of $U\left(H_{1}, H_{2}, \cdots, H_{k}\right)$. Therefore, each $H_{i}$ is a permutation graph. Assume that $U$ has smallest order among all such decompositions of $G$. We claim that $U$ is a prime permutation graph. Suppose that $U$ is not prime. Let

$$
U=V\left(L_{1}, L_{2}, \cdots, L_{p}\right)
$$

be a decomposition of $U$, where $V$ is non-trivial. Since $V$ is a decomposition of $U$ and vertices of $U$ are the induced subgraphs $H_{i}$ then each $L_{i}$ is a associated with subset of $\left\{H_{1}, H_{2}, \cdots, L_{k}\right\}$. We may assume that $L_{i}$ is an induced subgraph of $G$. Hence

$G=V\left(L_{1}, L_{2}, \cdots, L_{p}\right)$. But this contradicts the choice of $U$. Therefore, $U$ must be a prime permutation graph.

\section{Concluding Remarks}

Theorem 3.5 is a fair structural description of a per- 
mutation graph. Each $H_{i}$ in the decomposition

$$
G=U\left(H_{1}, H_{2}, \cdots, H_{k}\right)
$$

is a permutation graph and so is itself prime permutation graph or a composition of permutation graphs by a prime permutation graph. So we see that a permutation graph is expressible in terms of prime permutation graphs by compositions.

We have determined already the prime permutation trees, given in Theorem 3.4. One interesting problem to consider is the characterization of prime permutation graphs.

\section{REFERENCES}

[1] P. C. F. Ramos, "On Graphs of Inversions of Permutations,” Master's Thesis, University of the Philippines, Baguio City, 2012.

[2] S. Skiena, "Implementing Discrete Mathematics: Combinatorics and Graph Theory with Mathematica," AddisonWesley, Reading, 1990.

[3] T. Gallai, “Transitiv Orientierbare Graphen,” Acta Mathe- matica Academiae Scientiarum Hungarica, Vol. 18, No. 1-2, 1967, pp. 25-66. doi:10.1007/BF02020961

[4] A. Pnueli, A. Lempel and S. Even, "Transitive Orientation of Graphs and Identification of Permutation Graphs,” Canadian Journal of Mathematics, Vol. 23, No. 1, 1971, pp. 160-175. doi:10.4153/CJM-1971-016-5

[5] V. Limouzy, "Seidel Minor, Permutation Graphs and Combinatorial Properties,” In: Lecture Notes in Computer Science Volume 6506, Springer, Berlin, 2010, pp. 194205.

[6] F. Harary, "Graph Theory,” Addison-Wesley Publishing Company, Boston, 1969.

[7] J. W. Moon, "Topics on Tournaments," Holt, Rinehart and Winston, New York, 1968.

[8] S. V. Gervacio, “Tournament Score Sequences,” Annals of the New York Academy of Sciences, Vol. 576, Graph Theory and Its Applications, East and West: Proceedings of 1st China-USA International Graph Theory Conference, Jinan, China, June 1986, pp. 200-202.

[9] F. Harary and A. J. Schwenk, "The Number of Caterpillars,” Discrete Mathematics, Vol. 6, No. 4, 1973, pp. 359365. doi:10.1016/0012-365X(73)90067-8 